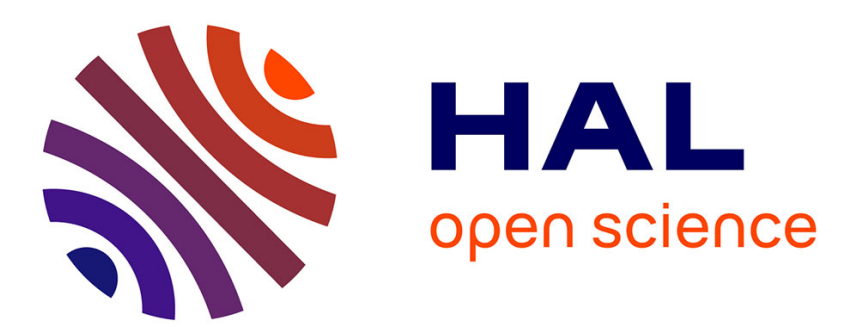

\title{
Lyapunov Functions for Shuffle Asymptotic Stability of Discrete-Time Switched Systems
}

\author{
Antoine Girard, Paolo Mason
}

\section{To cite this version:}

Antoine Girard, Paolo Mason. Lyapunov Functions for Shuffle Asymptotic Stability of DiscreteTime Switched Systems. IEEE Control Systems Letters, 2019, 3 (3), pp.499-504. 10.1109/LCSYS.2019.2909731 . hal-02286590

\section{HAL Id: hal-02286590 https://hal.science/hal-02286590}

Submitted on 13 Sep 2019

HAL is a multi-disciplinary open access archive for the deposit and dissemination of scientific research documents, whether they are published or not. The documents may come from teaching and research institutions in France or abroad, or from public or private research centers.
L'archive ouverte pluridisciplinaire HAL, est destinée au dépôt et à la diffusion de documents scientifiques de niveau recherche, publiés ou non, émanant des établissements d'enseignement et de recherche français ou étrangers, des laboratoires publics ou privés. 


\title{
Lyapunov Functions for Shuffle Asymptotic Stability of Discrete-Time Switched Systems
}

\author{
Antoine Girard ${ }^{1}$ and Paolo Mason ${ }^{1}$
}

\begin{abstract}
In this paper, we investigate stability of discretetime switched systems under shuffled switching signals. A switching signal is said to be shuffled if each mode of the switched system is activated infinitely often. We introduce the notion of shuffle Lyapunov functions and show that the existence of such a function is a sufficient condition for global uniform shuffle asymptotic stability. In the second part of the paper, we show that for a specific class of switched systems, with linear and invertible dynamics, existence of a shuffle Lyapunov function is also necessary, even for the weaker notion of global shuffle attractivity. Examples and numerical experiments are used to illustrate the main results of the paper.
\end{abstract}

Index Terms-Switched systems; Shuffle stability; Lyapunov methods

\section{INTRODUCTION}

Switched systems consist of a collection of dynamics with a switching rule specifying at each time instant the dynamics describing the evolution of the system. The popularity of switched systems is due to the fact that despite the simplicity of their mathematical formulation, they can exhibit complex and non-intuitive behavior. Moreover, their relevance in many engineering applications has motivated abundant research for almost three decades.

Early work on stability of switched systems has focused on stability for switching signals that are arbitrary or that satisfy some (minimum or average) dwell-time condition (see e.g. [1], [2], [3] and the references therein). More recently, several works have considered the problem of proving stability for subsets of switching signals. In general, such switching signals are assumed to be generated by some finite state automaton and stability is characterized either in term of constrained joint spectral radius [4], [5], [6] or using Lyapunov functions [7], [8], [9], [10]. However, there are some subsets of switching signals that cannot be specified using classical finite state automata. For instance, [11] considers switching signals specified using weighted-automata.

In this paper, we consider the class of discrete-time shuffled switching signals. A switching signal is said to be shuffled if each mode of the switched system is activated infinitely often. Since the sets of liveness and safety properties are disjoint [12], the set of shuffled switching signals, which is an example of liveness property, cannot be characterized using classical finite state automata that can only describe safety properties. Actually, shuffled switching signals may be seen as an example of $\omega$-regular language

\footnotetext{
${ }^{1}$ The authors are with Laboratoire des signaux et systèmes (L2S) CNRS, CentraleSupélec, Université Paris-Sud, Université ParisSaclay. 3, rue Joliot-Curie, 91192 Gif-sur-Yvette, cedex, France firstname. lastnamedl2s.centralesupelec.fr
}

which can be characterized using either non-deterministic Büchi automata, deterministic Rabin automata, or deterministic Muller automata, which are finite state automata with various acceptance conditions (see e.g. [12]). Shuffled switching signals have first been introduced in [13] for switched linear systems, where a necessary and sufficient condition for shuffle asymptotic stability (i.e. asymptotic stability with respect to shuffled switching signals) has been proposed based on the maximum spectral radius of an infinite set of matrices. Shuffled switching signals are also considered in [14], where it is shown that stability of a class of switched systems with switching signals constrained by an arbitrary $\omega$-regular language is equivalent to shuffle asymptotic stability of an associated lifted switched system. In the same paper, it is shown that some robust notion of shuffle asymptotic stability is equivalent to asymptotic stability for arbitrary switching signals.

Shuffled switching signals can also be related to multiagent consensus with switching communication topologies as follows. Consider that at each time instant only one edge in the communication graph is active and is selected by a switching signal. If one uses a shuffled switching signal, then each edge is activated infinitely often so that the union of future communication graphs is connected at all time, and it is well-known (see e.g. [15], [16]) that the consensus is asymptotically reached. On the contrary, if some edges are never activated the union of future communication graphs may be disconnected and the consensus cannot be approached.

In this work, we develop for the first time a Lyapunov approach to stability analysis for shuffled switching signals. In Section III, we introduce the notion of shuffle Lyapunov functions and show that the existence of such a function is a sufficient condition for global uniform shuffle asymptotic stability. In Section IV, we show that for a class of switched systems, with linear and invertible dynamics, existence of a shuffle Lyapunov function is also necessary, even for the weaker notion of global shuffle attractivity. Several examples are used to illustrate the main results of the paper. Finally, in Section V, a numerical example is provided.

Notations: In this paper, $\|$.$\| denotes the usual Eu-$ clidean norm on $\mathbb{R}^{n}$. A function $\gamma: \mathbb{R}_{0}^{+} \rightarrow \mathbb{R}_{0}^{+}$is a $\mathcal{K}$ function if it is continuous, strictly increasing and with $\gamma(0)=0$; it is a $\mathcal{K}_{\infty}$ function if it is a $\mathcal{K}$ function and additionally $\lim _{r \rightarrow+\infty} \gamma(r)=+\infty$. A function $\beta: \mathbb{R}_{0}^{+} \times$ $\mathbb{R}_{0}^{+} \rightarrow \mathbb{R}_{0}^{+}$is a $\mathcal{K} \mathcal{L}$ function if for all $s \in \mathbb{R}_{0}^{+}, \beta(., s)$ is a $\mathcal{K}$ function and for all $r \in \mathbb{R}_{0}^{+}, \beta(r,$.$) is non-increasing and$ $\lim _{s \rightarrow+\infty} \beta(r, s)=0$. 


\section{ShufFle Asymptotic Stability}

We consider a discrete-time switched system of the following form:

$$
x(t+1)=f_{\theta(t)}(x(t)), t \in \mathbb{N}
$$

where $x: \mathbb{N} \rightarrow \mathbb{R}^{n}$ is the trajectory and $\theta: \mathbb{N} \rightarrow \Sigma$ is the switching signal. $\Sigma=\{1, \ldots, m\}$, with $m \geq 2$, is the finite set of modes and $\left\{f_{\sigma}: \mathbb{R}^{n} \rightarrow \mathbb{R}^{n} \mid \sigma \in \Sigma\right\}$ is the collection of vector fields. Given an initial state $x_{0} \in \mathbb{R}^{n}$, and a switching signal $\theta$, the trajectory defined by (1) with $x(0)=x_{0}$ is unique and denoted $\mathbf{x}\left(., x_{0}, \theta\right)$.

In this paper, we are interested in a particular class of switching signals called shuffled. In words, a switching signal is said to be shuffled if each mode in $\Sigma$ is activated infinitely often. This translates formally as below:

Definition 1. A switching signal $\theta: \mathbb{N} \rightarrow \Sigma$ is shuffled if

$$
\forall \sigma \in \Sigma, \forall T \in \mathbb{N}, \exists t \geq T: \theta(t)=\sigma .
$$

The sequence of shuffling instants $\left(\tau_{k}^{\theta}\right)_{k \in \mathbb{N}}$ is defined by $\tau_{0}^{\theta}=0$ and for all $k \in \mathbb{N}$,

$$
\tau_{k+1}^{\theta}=\min \left\{t>\tau_{k}^{\theta} \mid \begin{array}{c}
\forall \sigma \in \Sigma, \exists s \in \mathbb{N}: \\
\tau_{k}^{\theta} \leq s<t \text { and } \theta(s)=\sigma
\end{array}\right\} .
$$

The shuffling index $\kappa^{\theta}: \mathbb{N} \rightarrow \mathbb{N}$ is given by

$$
\kappa^{\theta}(t)=\max \left\{k \in \mathbb{N} \mid \tau_{k}^{\theta} \leq t\right\} .
$$

Intuitively, between the instants $\tau_{k}^{\theta}$ and $\tau_{k+1}^{\theta}-1$, each mode in $\Sigma$ is activated at least once. For a shuffled switching signal, the sequence of shuffling instants is well-defined, strictly increasing and thus goes to $+\infty$. Similarly, the shuffling index is well-defined, non-decreasing and goes to $+\infty$. An illustration of these concepts is shown in Figure 1.

We study stability properties of switched system (1) under the effect of shuffled switching signals.

Definition 2. Switched system (1) is said to be

- globally shuffle attractive (GSA) if for all $x_{0} \in \mathbb{R}^{n}$, for all shuffled switching signal $\theta$ :

$$
\lim _{t \rightarrow+\infty}\left\|\mathbf{x}\left(t, x_{0}, \theta\right)\right\|=0
$$

- globally uniformly shuffle asymptotically stable (GUSAS) if there exists a $\mathcal{K} \mathcal{L}$ function $\beta$ such that for all $x_{0} \in \mathbb{R}^{n}$, for all shuffled switching signal $\theta$ :

$$
\left\|\mathbf{x}\left(t, x_{0}, \theta\right)\right\| \leq \beta\left(\left\|x_{0}\right\|, \kappa^{\theta}(t)\right), \forall t \in \mathbb{N} .
$$

We first establish some relations between stability notions:

Proposition 1. If switched system (1) is GUSAS, then it is $G S A$ and for all $x_{0} \in \mathbb{R}^{n}$, for all switching signal $\theta$ :

$$
\left\|\mathbf{x}\left(t, x_{0}, \theta\right)\right\| \leq \beta\left(\left\|x_{0}\right\|, 0\right), \forall t \in \mathbb{N} .
$$

Proof. If switched system (1) is GUSAS, then for all $x_{0} \in$ $\mathbb{R}^{n}$, for all shuffled switching signal $\theta$

$$
\lim _{t \rightarrow+\infty}\left\|\mathbf{x}\left(t, x_{0}, \theta\right)\right\| \leq \lim _{t \rightarrow+\infty} \beta\left(\left\|x_{0}\right\|, \kappa^{\theta}(t)\right)=0 .
$$


Fig. 1. A switching signal (with $m=2$ ) and the associated shuffling instants and index.

For the second part of the proposition, let $x_{0} \in \mathbb{R}^{n}$, and $\theta$ be a switching signal (not necessarily shuffled). Let $t \in \mathbb{N}$, it is clear that there exists a shuffled switching signal $\theta_{t}$, which coincides with $\theta$ up to time $t$. Then, we get

$$
\begin{aligned}
\left\|\mathbf{x}\left(t, x_{0}, \theta\right)\right\| & =\left\|\mathbf{x}\left(t, x_{0}, \theta_{t}\right)\right\| \\
& \leq \beta\left(\left\|x_{0}\right\|, \kappa^{\theta_{t}}(t)\right) \leq \beta\left(\left\|x_{0}\right\|, 0\right) .
\end{aligned}
$$

It is important to remark that the second part of Proposition 1 holds for all switching signals (not only shuffled). Then, if a switched system is GUSAS, it is also Lyapunov stable for all arbitrary switching signals. It is also clear that if a switched system is globally uniformly asymptotically stable for all switching signals then it is GUSAS. However, the converse is not true (see Example 1). In addition, a GSA switched system need not be GUSAS (see Example 2).

\section{SUFFICIENT CONDITIONS BASED ON LYAPUNOV FUNCTIONS}

In this section, we establish sufficient conditions for a switched system to be GUSAS based on the following notion of Lyapunov function:

Definition 3. A function $V: \Sigma \times \mathbb{R}^{n} \rightarrow \mathbb{R}_{0}^{+}$is called a shuffle Lyapunov function if there exists $\mathcal{K}_{\infty}$ functions $\alpha_{1}$, $\alpha_{2}$, and a $\mathcal{K}$ function $\rho$ with $\rho(r)<r$ for all $r>0$, such that for all $x \in \mathbb{R}^{n}$, the following hold:

$$
\begin{array}{lr}
\alpha_{1}(\|x\|) \leq V(\sigma, x) \leq \alpha_{2}(\|x\|), & \sigma \in \Sigma \\
V\left(\sigma, f_{\sigma^{\prime}}(x)\right) \leq V(\sigma, x), & \sigma, \sigma^{\prime} \in \Sigma, \sigma \neq \sigma^{\prime} \\
V\left(\sigma+1, f_{\sigma}(x)\right) \leq V(\sigma, x), & \sigma \in \Sigma \backslash\{m\} \\
V\left(1, f_{m}(x)\right) \leq \rho(V(m, x)) &
\end{array}
$$

Theorem 1. If there exists a shuffle Lyapunov function, then the switched system (1) is GUSAS.

Proof. Let us consider an initial condition $x_{0} \in \mathbb{R}^{n}$ and a shuffled switching signal $\theta$, let us denote $x()=.\mathbf{x}\left(., x_{0}, \theta\right)$. 


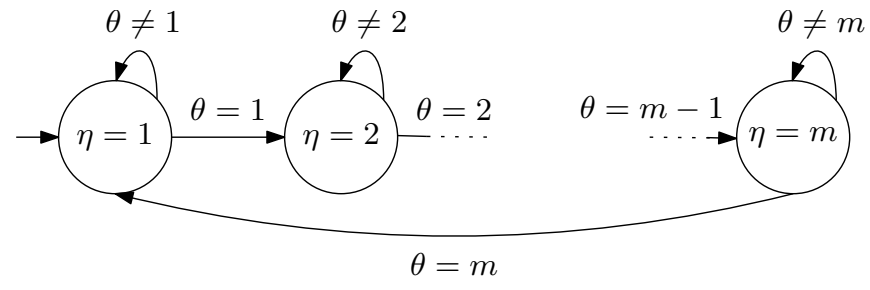

Fig. 2. Automaton describing the dynamics of $\eta$.

Let $\eta: \mathbb{N} \rightarrow \Sigma$ be defined by $\eta(0)=1$ and the following rules:

$$
\begin{cases}\text { if } \theta(t) \neq \eta(t), & \text { then } \eta(t+1)=\eta(t) ; \\ \text { if } \theta(t)=\eta(t) \text { and } \eta(t) \neq m, & \text { then } \eta(t+1)=\eta(t)+1 ; \\ \text { if } \theta(t)=\eta(t) \text { and } \eta(t)=m, & \text { then } \eta(t+1)=1 .\end{cases}
$$

Equivalently, the evolution of $\eta$ can be described by the finite state automaton shown on Figure 2. Now, let us consider $W: \mathbb{N} \rightarrow \mathbb{R}_{0}^{+}$, defined by $W(t)=V(\eta(t), x(t))$, for all $t \in \mathbb{N}$. According to the dynamics of $x$ and $\eta$, it follows from (3), (4) and (5) that for all $t \in \mathbb{N}, W(t+1) \leq W(t)$.

Let $k \in \mathbb{N}$, between the shuffling instants $\tau_{k}^{\theta}$ and $\tau_{k+1}^{\theta}-1$, there exists an instant $t$ such that $\theta(t)=\eta\left(\tau_{k}^{\theta}\right)$. Then, it follows that either $\eta\left(\tau_{k+1}^{\theta}\right) \geq \eta\left(\tau_{k}^{\theta}\right)+1$ or there exists an instant $t$ between $\tau_{k}^{\theta}$ and $\tau_{k+1}^{\theta}-1$ such that $\eta(t)=m$ and $\eta(t+1)=1$. From the previous statement, we can conclude that between the instants $\tau_{k}^{\theta}$ and $\tau_{k+m}^{\theta}-1$, there exists an instant $t$ such that $\eta(t)=m$ and $\eta(t+1)=1$, which from (5) gives that $W(t+1) \leq \rho(W(t))$. Using the fact that $W$ is non-increasing the rest of the time, we get for all $k \in \mathbb{N}$, $W\left(\tau_{k+m}^{\theta}\right) \leq \rho\left(W\left(\tau_{k}^{\theta}\right)\right)$. Hence, it follows that

$$
\forall i \in \mathbb{N}, W\left(\tau_{i \times m}^{\theta}\right) \leq \psi\left(W\left(\tau_{0}^{\theta}\right), i\right)=\psi(W(0), i)
$$

where $\psi: \mathbb{R}_{0}^{+} \times \mathbb{N} \rightarrow \mathbb{R}_{0}^{+}$is given for all $r \in \mathbb{R}_{0}^{+}$by $\psi(r, 0)=r$ and $\psi(r, i+1)=\rho(\psi(r, i))$, for $i \in \mathbb{N}$. Since $\rho$ is a $\mathcal{K}$ function, $\psi(., i)$ is also a $\mathcal{K}$ function for all $i \in \mathbb{N}$. Since $0 \leq \rho(s)<s$ for all $s>0$, we get that for all $r>0$, $\psi(r,$.$) is strictly decreasing and bounded below by 0$. By continuity of $\rho$ we get that $\lim _{i \rightarrow+\infty} \psi(r, i)=0$. Then, there exists a $\mathcal{K} \mathcal{L}$ function $\bar{\psi}: \mathbb{R}_{0}^{+} \times \mathbb{R}_{0}^{+} \rightarrow \mathbb{R}_{0}^{+}$such that for all $r \in \mathbb{R}_{0}^{+}$and $i \in \mathbb{N}, \bar{\psi}(r, i+1)=\psi(r, i)$.

Let $t \in \mathbb{N}$, and let $i \in \mathbb{N}$ such that $t \in\left[\tau_{i \times m}^{\theta}, \tau_{(i+1) \times m}^{\theta}\right)$, we get that $i \times m \leq \kappa^{\theta}(t)<(i+1) \times m$. Using monotonicity properties of $W$ and $\bar{\psi}$ and by (7), we have

$$
\begin{aligned}
W(t) & \leq W\left(\tau_{i \times m}^{\theta}\right) \leq \psi(W(0), i) \\
& \leq \bar{\psi}(W(0), i+1) \leq \bar{\psi}\left(W(0), \kappa^{\theta}(t) / m\right)
\end{aligned}
$$

Then, by (2), we get that for all $t \in \mathbb{N}$,

$$
\|x(t)\| \leq \alpha_{1}^{-1}\left(\bar{\psi}\left(\alpha_{2}(\|x(0)\|), \kappa^{\theta}(t) / m\right)\right)
$$

Let $\beta: \mathbb{R}_{0}^{+} \times \mathbb{R}_{0}^{+} \rightarrow \mathbb{R}_{0}^{+}$, be given for $r, s \in \mathbb{R}_{0}^{+}$by $\beta(r, s)=$ $\alpha_{1}^{-1}\left(\bar{\psi}\left(\alpha_{2}(r), s / m\right)\right)$. Using the properties of $\alpha_{1}, \alpha_{2}$ and $\bar{\psi}$, we get that $\beta$ is a $\mathcal{K} \mathcal{L}$ function.
The intuition of the proof of Theorem 1 is the following. The function $W$ is always non-increasing and is strictly decreasing when the value of $\eta$ transitions from $m$ to 1 . By construction of the dynamics of $\eta$, this happens an infinite number of times if and only if $\theta$ is a shuffled switching signal. Thus $W$ asymptotically converges towards 0 .

Remark 1. If $\alpha_{1}$ and $\alpha_{2}$ are quadratic functions $\left(\alpha_{1}(r)=\right.$ $\gamma_{1} r^{2}, \alpha_{2}(r)=\gamma_{2} r^{2}$, with $\left.0<\alpha_{1}<\alpha_{2}\right)$ and if $\rho$ is linear ( $\rho(r)=\lambda r$ with $0<\lambda<1$ ), then we get that $\beta$ is given by the closed form expression $\beta(r, s)=\sqrt{\frac{\gamma_{2}}{\gamma_{1} \lambda}} \lambda^{\frac{s}{2 m}} r$.

Example 1. Let us consider a switched system in $\mathbb{R}^{3}$ with 2 modes, where the vector fields are given by $f_{\sigma}(x)=A_{\sigma} x$ where

$$
\begin{aligned}
& A_{1}=\left(\begin{array}{ccc}
1 & 0 & 0 \\
0 & \lambda_{1} \cos \left(\phi_{1}\right) & -\lambda_{1} \sin \left(\phi_{1}\right) \\
0 & \lambda_{1} \sin \left(\phi_{1}\right) & \lambda_{1} \cos \left(\phi_{1}\right)
\end{array}\right), \phi_{1} \in[0,2 \pi], \lambda_{1} \in(0,1) \\
& A_{2}=\left(\begin{array}{ccc}
\lambda_{2} \cos \left(\phi_{2}\right) & -\lambda_{2} \sin \left(\phi_{2}\right) & 0 \\
\lambda_{2} \sin \left(\phi_{2}\right) & \lambda_{2} \cos \left(\phi_{2}\right) & 0 \\
0 & 0 & 1
\end{array}\right), \phi_{2} \in[0,2 \pi], \lambda_{2} \in(0,1) .
\end{aligned}
$$

It is clear that for unshuffled switching signals (i.e., in this case, switching signals that remain constant after some time), the state of the switched system will not converge to 0 asymptotically. To verify that the switched system is GUSAS, we use Theorem 1 with $V(1, x)=x_{1}^{2}+x_{2}^{2}+x_{3}^{2}$ and $V(2, x)=x_{1}^{2}+x_{2}^{2}+\frac{x_{3}^{2}}{\lambda_{1}}$. To simplify the exposition, we introduce the auxiliary function $U(x)=x_{1}^{2}+\frac{x_{2}^{2}}{\lambda_{1}}+\frac{x_{3}^{2}}{\lambda_{1}}$.

It is clear that (2) holds with $\alpha_{1}(r)=r$ and $\alpha_{2}(r)=\frac{r}{\lambda_{1}}$. Then, for (3), we check that

$$
V\left(1, A_{2} x\right)=\lambda_{2} x_{1}^{2}+\lambda_{2} x_{2}^{2}+x_{3}^{2} \leq V(1, x)
$$

and

$$
V\left(2, A_{1} x\right) \leq U\left(A_{1} x\right)=x_{1}^{2}+x_{2}^{2}+x_{3}^{3} \leq V(2, x) .
$$

From above, we also have that $V\left(2, A_{1} x\right) \leq V(1, x)$ and thus (4) holds. Finally, we also have

$$
V\left(1, A_{2} x\right) \leq \max \left(\lambda_{1}, \lambda_{2}\right) V(2, x) .
$$

Then, (5) holds with $\rho(r)=\max \left(\lambda_{1}, \lambda_{2}\right) r$. It follows that the switched system is GUSAS.

\section{A CONVERSE LYAPUNOV RESULT}

In this section, we identify a class of switched systems for which the sufficient conditions exposed in the previous section are also necessary for GUSAS. For this class of systems, we actually show an even stronger result, since we prove that if the switched system is GSA then there exists a shuffle Lyapunov function. This class is characterized by the following assumption:

Assumption 1. For all $\sigma \in \Sigma, f_{\sigma}(x)=A_{\sigma} x$ where $A_{\sigma}$ is an invertible matrix.

Before stating our main theorem, let us establish instrumental preliminary results. 


\section{A. Preliminary results}

Let $I \in \mathbb{R}^{n \times n}$ denote the identity matrix, given a finite sequence of matrices $M_{i} \in \mathbb{R}^{n \times n}, i=N_{1}, \ldots, N_{2}$ with $N_{1}, N_{2} \in \mathbb{N}, N_{1} \leq N_{2}$, we denote

$$
\prod_{i=N_{1}}^{N_{2}} M_{i}=M_{N_{2}} \times \cdots \times M_{N_{1}} .
$$

Then, we define several sets of matrices:

- $\mathcal{M}$ consists of all finite products of matrices in $\left\{A_{\sigma} \mid \sigma \in \Sigma\right\}$, together with the identity matrix;

- $\mathcal{M}_{\sigma}$ consists of all finite products of matrices in $\left\{A_{\sigma^{\prime}} \mid \sigma^{\prime} \in \Sigma \backslash\{\sigma\}\right\}$, for $\sigma \in \Sigma$, together with the identity matrix;

- $\mathcal{M}_{s}$ consists of all finite products of matrices in $\left\{A_{\sigma} \mid \sigma \in \Sigma\right\}$ where each matrix appears at least once.

Formally, we have:

$$
\begin{gathered}
\mathcal{M}=\left\{\prod_{i=0}^{N} A_{\sigma_{i}} \mid \begin{array}{c}
\sigma_{i} \in \Sigma, \\
i=0, \ldots, N, N \in \mathbb{N}
\end{array}\right\} \cup\{I\}, \\
\mathcal{M}_{\sigma}=\left\{\prod_{i=0}^{N} A_{\sigma_{i}} \mid \begin{array}{c}
\sigma_{i} \in \Sigma \backslash\{\sigma\}, \\
i=0, \ldots, N, N \in \mathbb{N}
\end{array}\right\} \cup\{I\}, \\
\mathcal{M}_{s}=\left\{\prod_{i=0}^{N} A_{\sigma_{i}} \mid \begin{array}{c}
\sigma_{i} \in \Sigma, i=0, \ldots, N, N \in \mathbb{N} \\
\text { and } \forall \sigma \in \Sigma, \exists i: \sigma_{i}=\sigma
\end{array}\right\} .
\end{gathered}
$$

Clearly, we have $\mathcal{M}_{\sigma} \subseteq \mathcal{M}$ for all $\sigma \in \Sigma$ and $\mathcal{M}_{s} \subseteq \mathcal{M}$.

The first two lemmas show that the convergence of trajectories towards 0 is equivalent to the convergence of the product of matrices towards 0 :

Lemma 1. Under Assumption 1, if the switched system (1) is GSA, then for all shuffled switching signal $\theta$ :

$$
\lim _{N \rightarrow+\infty} \prod_{i=0}^{N} A_{\theta(i)}=0 .
$$

Proof. Let $\theta$ be a shuffled switching signal, then

$$
\forall t \geq 1, \forall x_{0} \in \mathbb{R}^{n}, \mathbf{x}\left(t, x_{0}, \theta\right)=\prod_{i=0}^{t-1} A_{\theta(i)} x_{0} .
$$

Let us consider $\delta>0$ and let $e_{1}, \ldots, e_{n}$ denote the vectors of the canonical basis of $\mathbb{R}^{n}$. Since the system is GSA, there exists $T_{j}, j=1, \ldots, n$ such that

$$
\forall j=1, \ldots, n, \forall t \geq T_{j},\left\|\mathbf{x}\left(t, e_{j}, \theta\right)\right\| \leq \frac{\delta}{n} .
$$

Then, let $x_{0} \in \mathbb{R}^{n}$ with coordinates $x_{0,1}, \ldots, x_{0, n}$, let $T=$ $\max \left(T_{1}, \ldots, T_{n}\right)$, by linearity, it follows from above that

$$
\forall t \geq T,\left\|\mathbf{x}\left(t, x_{0}, \theta\right)\right\| \leq \sum_{i=1}^{n}\left|x_{0, j}\right|\left\|\mathbf{x}\left(t, e_{j}, \theta\right)\right\| \leq\left\|x_{0}\right\| \delta .
$$

Then by (8) we get that

$$
\forall t \geq T,\left\|\prod_{i=0}^{t-1} A_{\theta(i)}\right\| \leq \delta,
$$

which leads directly to the statement of Lemma 1.
The proof of the following result is straightforward from the previous lemma:

Lemma 2. Under Assumption 1, if the switched system (1) is GSA, then for all sequences of matrices $\left(M_{i}\right)_{i \in \mathbb{N}}$ with $M_{i} \in \mathcal{M}_{s}$ we have

$$
\lim _{N \rightarrow+\infty} \prod_{i=0}^{N} M_{i}=0 .
$$

Lemma 1 with Theorem 20 in [14] gives the following:

Lemma 3. Under Assumption 1, if the switched system (1) is GSA, then there exists $B>0$ such that for all $M \in \mathcal{M}$, $\|M\| \leq B$.

We finally get the following property:

Lemma 4. Under Assumption 1, if the switched system (1) is $G S A$, then there exist $\lambda \in(0,1)$ and a norm of $\mathbb{R}^{n}$ denoted $\|\cdot\|_{*}$ such that for all $M \in \mathcal{M}_{s},\|M\|_{*} \leq \lambda$.

Proof. By Lemma 3, we get that all matrices in $\mathcal{M}_{s}$ are bounded by $B$. Together with Lemma 2 , we get from Theorem 1 in [17], that the joint spectral radius of $\mathcal{M}_{s}$ is strictly less than 1 . Then, the conclusion is obtained by Lemma 2 in [17].

\section{B. Converse Lyapunov theorem}

We can now state the main result of the section:

Theorem 2. Under Assumption 1, the switched system (1) is GSA if and only if there exists a shuffle Lyapunov function.

Proof. The "if" part is a consequence of Theorem 1 and Proposition 1. The proof of the "only if" part is as follows. Let $\|.\|_{*}$ be as in Lemma 4 . Since all norms on $\mathbb{R}^{n}$ are equivalent, there exist $0<\kappa_{1}<\kappa_{2}$ such that for all $x \in \mathbb{R}^{n}$, $\kappa_{1}\|x\| \leq\|x\|_{*} \leq \kappa_{2}\|x\|$

Let $V: \Sigma \times \mathbb{R}^{n} \rightarrow \mathbb{R}_{0}^{+}$be defined for $\sigma \in \Sigma \backslash\{m\}$ by

$$
V(\sigma, x)=\sup _{\substack{M_{m} \in \mathcal{M}_{m}, M_{m-1} \in \mathcal{M}_{m-1}, M_{\sigma} \in \mathcal{M}_{\sigma}}}\left\|M_{m}\left(\prod_{i=\sigma}^{m-1} A_{i} M_{i}\right) x\right\|_{*},
$$

and

$$
V(m, x)=\sup _{M_{m} \in \mathcal{M}_{m}}\left\|M_{m} x\right\|_{*} .
$$

Let us prove that $V$ is a shuffle Lyapunov function.

Since $I$ belongs to $\mathcal{M}_{\sigma}$ for all $\sigma \in \Sigma$ we get for all $\sigma \in \Sigma \backslash\{m\}$,

$$
V(\sigma, x) \geq\left\|\left(\prod_{i=\sigma}^{m-1} A_{i}\right) x\right\|_{*} \geq \kappa_{1}\left\|\left(\prod_{i=\sigma}^{m-1} A_{i}\right) x\right\|
$$

and $V(m, x) \geq\|x\|_{*} \geq \kappa_{1}\|x\|$. Since all matrices $A_{\sigma}$ are invertible it follows that there exists $\gamma_{1}>0$ such that $V(\sigma, x) \geq \gamma_{1}\|x\|$ for all $\sigma \in \Sigma$, for all $x \in \mathbb{R}^{n}$. From (9) and (10), and from Lemma 3, we get that for all $\sigma \in \Sigma$, for all $x \in \mathbb{R}^{n}$

$$
V(\sigma, x) \leq \sup _{M \in \mathcal{M}}\|M x\|_{*} \leq \kappa_{2} \sup _{M \in \mathcal{M}}\|M x\| \leq \kappa_{2} B\|x\| .
$$


Hence, (2) holds.

Let $\sigma, \sigma^{\prime} \in \Sigma$ with $\sigma \neq \sigma^{\prime}$. If $\sigma \neq m$,

$$
V\left(\sigma, A_{\sigma^{\prime}} x\right)=\sup _{\substack{M_{m} \in \mathcal{M}_{m}, M_{m-1} \in \mathcal{M}_{m-1}, M_{\sigma} \in \mathcal{M}_{\sigma}}}\left\|M_{m}\left(\prod_{i=\sigma}^{m-1} A_{i} M_{i}\right) A_{\sigma^{\prime}} x\right\|_{*} .
$$

For all $M_{\sigma} \in \mathcal{M}_{\sigma}, M_{\sigma} A_{\sigma^{\prime}} \in \mathcal{M}_{\sigma}$ and therefore it follows that $V\left(\sigma, A_{\sigma^{\prime}} x\right) \leq V(\sigma, x)$. If $\sigma=m$,

$$
V\left(m, A_{\sigma^{\prime}} x\right)=\sup _{M_{m} \in \mathcal{M}_{m}}\left\|M_{m} A_{\sigma^{\prime}} x\right\|_{*} .
$$

For all $M_{m} \in \mathcal{M}_{m}, M_{m} A_{\sigma^{\prime}} \in \mathcal{M}_{m}$ and therefore it follows that $V\left(m, A_{\sigma^{\prime}} x\right) \leq V(m, x)$. Hence (3) holds.

Let $\sigma \in \Sigma \backslash\{m\}$. If $\sigma \neq m-1$, then

$$
V\left(\sigma+1, A_{\sigma} x\right)=\sup _{\substack{M_{m} \in \mathcal{M}_{m}, M_{m-1} \in \mathcal{M}_{m-1}, M_{\sigma+1} \in \mathcal{M}_{\sigma+1}}}\left\|M_{m}\left(\prod_{i=\sigma+1}^{m-1} A_{i} M_{i}\right) A_{\sigma} x\right\|_{*} .
$$

Since $I \in \mathcal{M}_{\sigma}$, it follows that $V\left(\sigma+1, A_{\sigma} x\right) \leq V(\sigma, x)$. If $\sigma=m-1$, then

$$
V\left(\sigma+1, A_{\sigma} x\right)=\sup _{M_{m} \in \mathcal{M}_{m}}\left\|M_{m} A_{m-1} x\right\|_{*} .
$$

Since $I \in \mathcal{M}_{m-1}$, it follows that $V\left(\sigma+1, A_{\sigma} x\right) \leq V(\sigma, x)$. Hence (4) holds.

Finally, from Lemma 4,

$$
\begin{aligned}
V\left(1, A_{m} x\right) & =\sup _{\substack{M_{m} \in \mathcal{M}_{m}, M_{m-1} \in \mathcal{M}_{m-1}, M_{1} \in \mathcal{M}_{1}}}\left\|M_{m}\left(\prod_{i=1}^{m-1} A_{i} M_{i}\right) A_{m} x\right\|_{*} \\
& \leq \sup _{M \in \mathcal{M}_{s}}\|M x\|_{*} \leq \lambda\|x\|_{*} \leq \lambda V(m, x) .
\end{aligned}
$$

Hence (5) holds and $V$ is a shuffle Lyapunov function.

Corollary 1. Under Assumption 1, the switched system (1) is GSA if and only if it is GUSAS.

Proof. The "if" part is a consequence of Proposition 1. For the "only if" part, if the switched system (1) is GSA then from Theorem 2, there exists a shuffle Lyapunov function, and from Theorem 1, the switched system is GUSAS.

Example 2. The following example shows that by removing Assumption 1, the converse Lyapunov result stated in Theorem 2 does not hold anymore. Let us consider a switched system in $\mathbb{R}^{2}$ with 2 modes, where the vector fields are given by $f_{\sigma}(x)=A_{\sigma} x$ where

$$
A_{1}=\left(\begin{array}{ll}
0 & 0 \\
0 & 2
\end{array}\right), A_{2}=\left(\begin{array}{ll}
2 & 0 \\
0 & 0
\end{array}\right) .
$$

Clearly, the switched system is GSA since after both modes have been activated, the state of the system is equal to 0 for all time. We proceed by contradiction. Let us assume that there exists a shuffle Lyapunov function, then by Theorem 1 the switched system is GUSAS. Hence, there exists a $\mathcal{K} \mathcal{L}$ function $\beta$ such that for all $x_{0} \in \mathbb{R}^{2}$, for all shuffled switching signal $\theta$ :

$$
\left\|\mathbf{x}\left(t, x_{0}, \theta\right)\right\| \leq \beta\left(\left\|x_{0}\right\|, \kappa^{\theta}(t)\right), \forall t \in \mathbb{N} .
$$

Consider $x_{0}=\left(\begin{array}{l}0 \\ 1\end{array}\right)$, and for $t \in \mathbb{N}$ let $\theta_{t}$ be a shuffled switching signal such that for all $s=0, \ldots, t, \theta_{t}(s)=1$. Let us remark that $\mathbf{x}\left(t+1, x_{0}, \theta_{t}\right)=\left(\begin{array}{c}0 \\ 2^{t}\end{array}\right)$ while $\left\|x_{0}\right\|=1$ and $\kappa^{\theta_{t}}(t+1)=0$. Hence, we should have for all $t \in \mathbb{N}$, $2^{t} \leq \beta(1,0)$, which yields a contradiction.

Remark 2. While the invertibility of the matrices $A_{\sigma}$, $\sigma \in \Sigma$ is not used in the proofs for Lemmas 1 and 2, it is crucial for Lemmas 3 and 4 (see the proof of Theorem 20 in [14]). However, when the switched system is GUSAS, the conclusion of Lemma 3 (and thus of Lemma 4 and Theorem 2) holds without the invertibility assumption. Thus, it follows from Theorem 1, that a switched linear system is GUSAS if and only if it admits a shuffle Lyapunov function.

\section{NumericAl EXAMPLE}

As an illustration of the results of the paper, we consider a synchronization problem. We consider a multi-agent system consisting of three discrete-time oscillators whose dynamics is given by

$$
z_{i}(t+1)=R z_{i}(t)+u_{i}(t), i=1, \ldots, 3
$$

where $z_{i}(t) \in \mathbb{R}^{2}, u_{i}(t) \in \mathbb{R}^{2}$ and $R=\left(\begin{array}{cc}\cos (\phi) & -\sin (\phi) \\ \sin (\phi) & \cos (\phi)\end{array}\right)$ with $\phi=\frac{\pi}{6}$. The input $u_{i}(t)$ is used for synchronization purpose and is based on the available information at time $t$. There exist two communication channels: channel 1 between agents 1 and 2 and channel 2 between agents 2 and 3 . At each instant, only one of these channels is active and the active channel is selected by a switching signal $\theta: \mathbb{N} \rightarrow$ $\Sigma=\{1,2\}$. Then, the input value is given as follows

$$
\begin{aligned}
& u_{1}(t)= \begin{cases}\gamma\left(z_{2}(t)-z_{1}(t)\right), & \text { if } \theta(t)=1 \\
0 & \text { if } \theta(t)=2\end{cases} \\
& u_{2}(t)= \begin{cases}\gamma\left(z_{1}(t)-z_{2}(t)\right), & \text { if } \theta(t)=1 \\
\gamma\left(z_{3}(t)-z_{2}(t)\right), & \text { if } \theta(t)=2\end{cases} \\
& u_{3}(t)= \begin{cases}0, & \text { if } \theta(t)=1 \\
\gamma\left(z_{2}(t)-z_{3}(t)\right), & \text { if } \theta(t)=2\end{cases}
\end{aligned}
$$

where $\gamma=0.1$. Denoting the vector of synchronization errors as $x(t)=\left(\begin{array}{c}z_{2}(t)-z_{1}(t) \\ z_{3}(t)-z_{2}(t)\end{array}\right)$, the error dynamics is described by a 4-dimensional switched linear system with 2 modes given by the following matrices where $I_{2}$ is the 2 dimensional identity matrix:

$$
A_{1}=\left(\begin{array}{cc}
R-2 \gamma I_{2} & 0 \\
\gamma I_{2} & R
\end{array}\right), A_{2}=\left(\begin{array}{cc}
R & \gamma I_{2} \\
0 & R-2 \gamma I_{2}
\end{array}\right) .
$$

We want to show that the agents synchronize if both communication channels are activated infinitely often. This can be done by verifying that the error system is GUSAS. We then look for a shuffle Lyapunov function of the form $V(\sigma, x)=x^{\top} Q_{\sigma} x$ where $Q_{\sigma}$ is a positive definite symmetric matrix. For this form of function, for a linear function $\rho$ given by $\rho(r)=\lambda r$ with $\lambda \in(0,1)$, the conditions in Theorem 1 translate directly into the following linear matrix inequalities:

$$
\begin{array}{rlrl}
I & \leq Q_{\sigma}, & \sigma \in \Sigma \\
A_{\sigma^{\prime}}^{\top} Q_{\sigma} A_{\sigma^{\prime}} & \leq Q_{\sigma} & \sigma, \sigma^{\prime} \in \Sigma, \sigma \neq \sigma^{\prime} \\
A_{\sigma}^{\top} Q_{\sigma+1} A_{\sigma} & \leq Q_{\sigma}, & \sigma \in \Sigma \backslash\{m\} \\
A_{m}^{\top} Q_{1} A_{m} & \leq \lambda Q_{m} . & &
\end{array}
$$



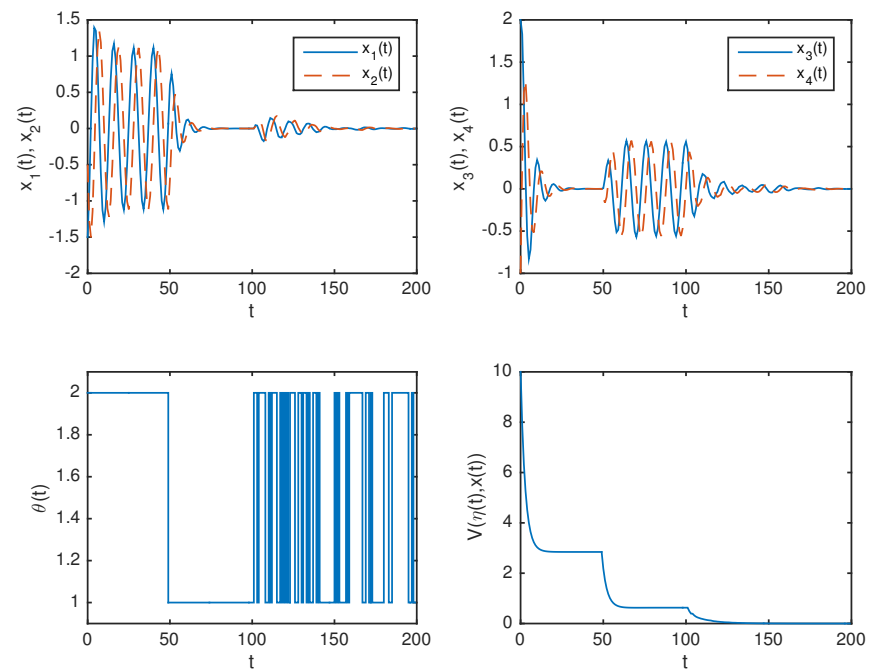

Fig. 3. Time evolution of the synchronization error $x(t)$ (top figures), switching signal $\theta(t)$ (bottom left), and shuffle Lyapunov function $V(\eta(t), x(t))$ (bottom right).

Solving these numerically, we find for $\lambda=0.88$ :

$$
Q_{1}=\left(\begin{array}{ll}
2.28 I_{2} & 1.14 I_{2} \\
1.14 I_{2} & 2.01 I_{2}
\end{array}\right), Q_{2}=\left(\begin{array}{ll}
2.00 I_{2} & 1.02 I_{2} \\
1.02 I_{2} & 2.05 I_{2}
\end{array}\right)
$$

Hence, the system is GUSAS.

For illustration, we consider the following scenario: for the first 50 time units, communication channel 2 is constantly activated, then at $t=50$ a switch occurs and for the next 50 time units communication channel 1 is constantly activated, after $t=100$ the communication are activated randomly with equal probability so that the switching signal is shuffled with probability 1 . The simulation results are shown in Figure 3. It is interesting to remark that when the switching signal remains constant the synchronization error does not go to zero. Indeed, before $t=50$, agents 2 and 3 synchronize but not agents 1 and 2; and between $t=50$ and $t=100$, agents 1 and 2 synchronize but agents 2 and 3 desynchronize. After $t=100$, the switching signal starts to shuffle and one can see that all agents achieve synchronization. Similar observations can be made on the evolution of the shuffle Lyapunov function $V(\eta(t), x(t))$ where $\eta(t)$ is defined as in the proof of Theorem 1 . As expected, this function is always non-increasing. When the switching signal remains constant, between $t=0$ and $t=49$, and between $t=50$ and $t=99$, one can see that the Lyapunov function reaches a constant value, which is not zero. As soon as the switching signal starts to shuffle, the Lyapunov function approaches 0.

\section{CONCLUSION}

In this paper, we have established several stability results for discrete-time switched systems with shuffled switching signals. The main contribution of the paper consists in a sufficient condition for global uniform asymptotic stability given by the existence of a shuffle Lyapunov function. For a class of switched linear systems, this condition is also shown to be necessary, even for the weaker notion of global shuffle attractivity. We have shown on a numerical example, how shuffle Lyapunov functions can be effectively computed using linear matrix inequalities.

The current work opens several research directions for the future. First, the development of numerical techniques to compute shuffle Lyapunov functions is necessary for cases where the simple linear matrix inequalities approach used in the paper is not successful. Secondly, for switched linear systems, it should be possible to define a joint spectral radius related to shuffled switching signals. It would be interesting to investigate its properties and to develop algorithms for its approximation. Finally, as shuffle stability seems to be related to stability with $\omega$-regular switching sequences, it would be interesting to develop a Lyapunov approach for switched systems with switching signals specified by some $\omega$-regular language.

\section{REFERENCES}

[1] Z. Sun and S. S. Ge, Switched linear systems: control and design. Springer, 2006.

[2] D. Liberzon, Switching in systems and control. Springer Science \& Business Media, 2003.

[3] H. Lin and P. J. Antsaklis, "Stability and stabilizability of switched linear systems: a survey of recent results," IEEE Transactions on Automatic control, vol. 54, no. 2, pp. 308-322, 2009.

[4] X. Dai, "A Gel'fand-type spectral radius formula and stability of linear constrained switching systems," Linear Algebra and its Applications, vol. 436, no. 5, pp. 1099-1113, 2012.

[5] V. Kozyakin, "The Berger-Wang formula for the Markovian joint spectral radius," Linear Algebra and its Applications, vol. 448, pp. $315-328,2014$

[6] X. Xu and B. Acikmese, "Approximation of the constrained joint spectral radius via algebraic lifting," arXiv preprint arXiv:1807.03965, 2018.

[7] J.-W. Lee and G. E. Dullerud, "Uniformly stabilizing sets of switching sequences for switched linear systems," IEEE Transactions on Automatic Control, vol. 52, no. 5, pp. 868-874, 2007.

[8] N. Athanasopoulos and M. Lazar, "Stability analysis of switched linear systems defined by graphs," in IEEE Conference on Decision and Control, 2014, pp. 5451-5456.

[9] M. Philippe, R. Essick, G. E. Dullerud, and R. M. Jungers, "Stability of discrete-time switching systems with constrained switching sequences," Automatica, vol. 72, pp. 242-250, 2016.

[10] P. Pepe, "Converse Lyapunov theorems for discrete-time switching systems with given switches digraphs," IEEE Transactions on Automatic Control, 2018.

[11] A. Kundu and D. Chatterjee, "On stability of discrete-time switched systems," Nonlinear Analysis: Hybrid Systems, vol. 23, pp. 191-210, 2017.

[12] C. Baier and J.-P. Katoen, Principles of model checking. MIT press, 2008.

[13] L. Gurvits, "Stability of discrete linear inclusion," Linear algebra and its applications, vol. 231, pp. 47-85, 1995.

[14] Y. Wang, N. Roohi, G. E. Dullerud, and M. Viswanathan, "Stability analysis of switched linear systems defined by regular languages." IEEE Transactions on Automatic Control, vol. 62, no. 5, pp. 25682575, 2017.

[15] L. Moreau, "Stability of multiagent systems with time-dependent communication links," IEEE Transactions on automatic control, vol. 50, no. 2, pp. 169-182, 2005.

[16] V. D. Blondel, J. M. Hendrickx, A. Olshevsky, and J. N. Tsitsiklis, "Convergence in multiagent coordination, consensus, and flocking," in IEEE Conference on Decision and Control, 2005, pp. 2996-3000.

[17] M. A. Berger and Y. Wang, "Bounded semigroups of matrices," Linear Algebra and its Applications, vol. 166, pp. 21-27, 1992. 